\title{
Changing infant death rates: diagnostic shift, success story, or both?
}

\author{
Roger W. Byard
}

Accepted: 14 May 2012/Published online: 5 June 2012

(C) Springer Science+Business Media, LLC 2012

Over the past 20 years there have been remarkable and gratifying falls in the rates of infant deaths in many Western countries due to declining numbers of cases attributed to sudden infant death syndrome (SIDS). This reduction in unexpected infant fatalities has been due in large part to the effects of the "Reduce the Risks" campaigns where community awareness programs were undertaken by local and national SIDS organizations to warn parents and carers of the potential dangers of prone sleeping, cigarette smoke exposure, head covering and overheating [1]. However, in the late 1970s changes in infant mortality had also been noted in a number of jurisdictions which were not due to a real reduction in lethal events, but were due instead to pathologists reassigning causes of death-in this instance from respiratory infections to SIDS [2]. This was termed a diagnostic shift and was clearly identified by a lack of change in overall infant mortality figures. Diagnostic shift was regarded as an unfortunate event which had the potential to influence the recording of mortality figures.

Concerns were expressed that the fall in infant death rates in the early 1990s might be due to a similar diagnostic shift distorting mortality data [3, 4]. In this instance, however, studies revealed that the fall in numbers of SIDS deaths was matched by an associated fall in overall infant mortality, and that other causes of infant death had not significantly increased [4]. Thus, changing diagnostic practice by pathologists was not thought to be a factor in the initial change in patterns of infant death.

\section{R. W. Byard $(\square)$}

Discipline of Anatomy and Pathology, Level 3 Medical School

North Building, The University of Adelaide, Frome Road,

Adelaide 5005, Australia

e-mail: roger.byard@sa.gov.au
In addition to identifying risk factors for SIDS, the 1990s was also characterized by moves to improve infant death investigations with the establishment of standardized protocols for both death scene and autopsy examinations $[5,6]$. These protocols were aimed at improving diagnostic accuracy by providing additional information to enable more accurate determination of causes of death and to facilitate more meaningful comparisons of infant death rates between populations. In addition it was hoped that international guidelines would improve research in this area and further reduce infant death rates. The International Standardized Autopsy Protocol (ISAP) and Sudden Unexplained Infant Death Investigation Report Form (SUIDIRF) were subsequently formulated, endorsed by professional bodies, and published [5, 6].

Trends in infant deaths and diagnostic practices continued to be monitored throughout the 1990s and this subsequently showed that changes in diagnoses were occurring; i.e. the concerns that a diagnostic shift was present were realized. Pathologists had been identifying more cases of accidental asphyxia from dangerous sleeping environments $[7,8]$, in addition to detecting rarer forms of sudden death involving underlying metabolic and genetic conditions [9]. Although this was initially considered to be a problem, representing again a possible arbitrary reclassification of deaths, it was soon realized that this was in fact a positive finding. Changes in diagnoses in this instance endorsed the usefulness of protocols by demonstrating that they were providing useful information to help pathologists to identify conditions that would previously have been included under the umbrella of "SIDS." In other words, protocols were allowing more cases of unexpected infant death to be removed from the "diagnostic dustbin" of SIDS [10].

As is often the case, however, improvements in medicine are often accompanied by opposing trends. In this case 
a component of the diagnostic shift was identified which did involve subjective reassignment of causes of death. A trend had emerged in which some pathologists had moved away from using the term SIDS in favor of terms such as "unascertained" or "undetermined," or of "asphyxia" in all cases of shared sleeping [11]. There were many reasons for this change which included dissatisfaction with using a term such as SIDS in the absence of diagnostic features [12], and concerns that the term SIDS may obscure cases of accidental or inflicted suffocation. Individual pathologists and institutions were sometimes using terminology that was idiosyncratic and different to that used by others, something that is not good practice.

While it is clear that the fall in infant death rates due to SIDS over the past two decades has been genuine, cases have certainly been reassigned to other categories. One way of approaching this issue is to use the term sudden unexpected death in infancy (SUDI) or sudden unexpected infant death (SUID) [11] as this captures all infant deaths, although it also raises problems with central coding of data. However, subsequent analyses of data can be used to dissect out subcategories of cases using standard criteria. So, in the end, although there has been a genuine fall in infant mortality, there has also been a diagnostic shift. Part of the shift has been due to an improvement in diagnostic approaches, and part has been due merely to alterations in terminology. The only way to deal with these variables when interpreting mortality rates is to constantly monitor trends and to be vigilant in defining how cases have been diagnosed and classified. Unfortunately the SIDS literature is littered with papers that have not clearly specified definitions and diagnostic criteria, making interpretations of trends and research findings difficult [13]. The same problems occur with national mortality databases and must be recognized when analyses are being undertaken or interpreted.

\section{References}

1. Tursan d'Espaignet E, Bulsara M, Wolfenden L, Byard RW, Stanley FJ. Trends in sudden infant death syndrome in Australia from 1980-2002. Forensic Sci Med Pathol. 2008;4:83-90.

2. Byard RW. Sudden infant death syndrome. In: Byard RW, editor. Sudden death in the young. 3rd ed. Cambridge, UK: Cambridge University Press; 2010.

3. Malloy MH, MacLorman M. Changes in the classification of sudden unexpected infant deaths: United States, 1992-2001. Pediatrics. 2005;115:1247-53.

4. Byard RW, Beal SM. Has changing diagnostic preference been responsible for the recent fall in incidence of sudden infant death syndrome in South Australia? J Paediatr Child Health. 1995;31: 197-9.

5. http://www.cdc.gov/SIDS. Accessed 10 May 2012.

6. Krous HF. An international standardised autopsy protocol for sudden unexpected infant death. In: Rognum TO, editor. Sudden infant death syndrome. New trends in the nineties. Oslo: Scandinavian University Press; 1995. p. 81-95.

7. Beal SM, Byard RW. Accidental death or sudden infant death syndrome? J Paediatr Child Health. 1995;31:269-71.

8. Byard RW. Accidental childhood death and the role of the pathologist. Pediatr Dev Pathol. 2000;3:405-18.

9. Mitchell E, Krous HF, Donald T, Byard RW. Changing trends in the diagnosis of sudden infant death. Am J Forensic Med Pathol. 2000;21:311-4.

10. Emery JL. Is sudden infant death syndrome a diagnosis? Or is it just a diagnostic dustbin? Brit Med J. 1989;299:1240.

11. Byard RW. SUDI or "undetermined"-does it matter? Forensic Sci Med Pathol. 2009;5:252-3.

12. Byard RW. Possible mechanisms responsible for the sudden infant death syndrome. J Paediatr Child Health. 1991;27:147-57.

13. Byard RW, Lee V. A re-audit of the use of definitions of sudden infant death syndrome (SIDS) in peer-reviewed journals. J Forensic Leg Med. 2012;. doi:10.1016/j.jflm.2012.04.004. 